\title{
¿El Fondo Monetario Internacional y el Banco Mundial necesitan reformas?
}

Dahiam Pinzón Ramírez ${ }^{15}$

Recibido: 1 de noviembre 2007 Aprobado: 29 de noviembre 2007.

Pinzón R., D. (2007) ¿El Fondo Monetario Internacional y el Banco Mundial necesitan reformas? Revista Activos, 13, 57-68.

\section{Resumen}

El Fondo Monetario Internacional y el Banco Mundial son organismos creados para generar un equilibrio, tanto financiero como social, en todos los países del mundo. Pero, con el pasar de los años estas entidades han venido cambiando su manera de actuar, por la gran presión que ejerce el Grupo de los Siete, especialmente, Estados Unidos, razón por la cual, es de vital importancia buscar alternativas que den solución a las falencias que presentan estos organismos, dado que su intervención en las crisis de México en 1995, Asia del Este en 1997 y Rusia en 1998, derivó en condiciones que empeoraron y contribuyeron a que las crisis financieras se convirtieran en depresiones sociales y económicas que generaron catástrofes, lo cual cuestiona su papel en el contexto de la arquitectura financiera internacional actualmente.

Palabras clave: Fondo Monetario Internacional (FMI), Banco Mundial (BM), El Grupo del Banco Mundial, Nueva Arquitectura Financiera Internacional (NAFI), E1 Grupo de los Siete (G7), Imperio. JEL: F01, F02.

Pinzón R., D. (2007). Do the International Monetary Fund and the World Bank need reforms?. Activos Review, 13, 57-68.

\footnotetext{
${ }^{15}$ Estudiante de Contaduría Pública de la Universidad Santo Tomás, Sede principal, tumida_8956@hotmail.com.
} 
ACTIVOS | REVISTA de la FaCultad de contaduría pública

\begin{abstract}
The International Monetary Fund and the World Bank are organisms created to generate a balance, both financial and social, in all the countries of the world. But, with spending of the years these entities have come changing his way of operating, for the big pressure that the Group of the Seven, especially the United States; reason for which, it is of vital importance to look for alternatives that give solution to the bankruptcies that these organisms present since due to the intervention they take part in the crises of Mexico in 1995, East Asia in 1997 and Russia in 1998, derived in conditions that deteriorated and contributed that the financial crises were turning into social and economic depressions that generated catastrophes, which questions its role in the context of the international financial architecture at present momento.
\end{abstract}

Key words: International Monetary Fund (IMF), World Bank (BM), The Group of the World Bank, New International Financial Architecture (NAFI), The Group of Seven (G7), Empire. JEL: F01, F02.

\title{
Pinzón R., D. (2007). Le Fond Monétaire International et la Banque Mondiale ont-ils besoin des réformes?. Revue Activos, 13, 57-68.
}

\section{Résumé}

Le Fonds Monétaire International et la Banque mondiale sont des organismes créés pour établir un équilibre financier et social, dans tous les pays du monde. Mais, au fil des années ces organismes ont Changé leur façon d'agir, grâce à la pression qu'exercent le G7 et les ÉtatsUnis en particulier. C'est la raison pour laquelle il est important de chercher des alternatives qui donnent des solutions aux erreurs commises par ces organismes, étant donné que leur intervention dans les crises du Mexique en 1995, l'Asie de l'Est en 1997 et la Russie en 1998, a dérivé dans les conditions qui ont empiré et contribué à ce que les crises financières se convertissent en dépressions sociales et économiques. Ce qui a engendré des catastrophes, et qui met en question son rôle dans le contexte actuel de l'architecture financière internationale.

Mots clé: Le Fond Monétaire International (FMI), La Banque Mondiale (BM), Groupe de la Banque Mondiale, la Nouvelle Architecture Financière Internationale (NAFI), Le G7 « Le Groupe des Sept». Imperio JEL: F01, F02. 


\section{Introducción}

En la época del esplendor democrático de Atenas, una de cada diez personas tenía derechos ciudadanos. Veinticinco siglos después, es evidente que los griegos eran muy generosos, ya que lo que se evidencia hoy día en el desarrollo mundial es la hegemonía de los países desarrollados sobre los países en desarrollo.

Esto ha condicionado el actuar de organismos multilaterales que aunque han surgido para ayudar a los países en situaciones de crisis, las grandes potencias no permiten cumplir a cabalidad esta función. En este sentido, ha surgido la propuesta de replantear la arquitectura financiera internacional, con el fin de establecer estrategias y mecanismos que permitan mitigar esta situación.

Lo planteado propone estudiar y analizar la situación que se presenta con organismos como el Fondo Monetario Internacional y el Banco Mundial. Para ello, en el presente escrito se realiza una descripción sobre las características y funciones de estos dos organismos. Posteriormente, se analizan los inconvenientes que tienen estas dos instituciones para cumplir a satisfacción con sus objetivos. Y por último, a partir de lo analizado, se plantea una serie de alternativas que permitan mejorar los objetivos del Fondo Monetario Internacional y del Banco Mundial.

\section{Nacimiento de dos de los más grandes organismos mundiales para el equilibrio financiero y la reducción de la pobreza}

Tanto el Fondo Monetario Internacional como el Banco Mundial fueron creados en julio de 1944 en Bretton Woods (New Hampshire, USA) cuando se empezaba a terminar la II Guerra Mundial y a puertas de iniciar la Guerra Fría. En un principio fueron configurados para apoyar a los países desarrollados en los momentos de crisis cuando se presentaran desequilibrios en sus sistemas financieros, por un lado, y ayudar a las víctimas o más bien a los damnificados por las guerras que fueron, habían o se desarrollarían con el pasar de los años, por el otro. 
ACTIVOS | REVISTA de la FaCultad de contaduría pública

Es de vital importancia destacar que el Fondo Monetario se originó para tener un sistema monetario global que fuese fiable y en el cual la economía en todo el planeta entrara de la misma manera.

Éste debía hacer que reinara la paz y por eso tenía que mantener el control en las políticas monetarias, la estabilidad de los mercados y en especial ayudar a las naciones cuyas economías entraran en un gran retroceso y quietud.

El Fondo Monetario Internacional sería el encargado de gestionar el nuevo sistema monetario basado en el patrón divisas-oro, cumpliendo a la vez una función reguladora y crediticia. La función crediticia tenía que contribuir a evitar la devaluación de una moneda de cara a desequilibrios transitorios de la balanza de pagos de un país, mediante préstamos a corto plazo.

La función reguladora registraba las paridades ${ }^{16}$ de cada moneda con el oro y el dólar, tratando de evitar variaciones de los tipos de cambio hacia el futuro, con el fin de facilitar el desarrollo del comercio mundial; también debía supervisar la evolución monetaria en el mundo y de cada país miembro (Fondo Monetario Internacional, 2001).

De otro lado, el Banco Mundial se creó con el propósito de reducir la pobreza, mediante préstamos de bajo interés, créditos sin intereses a nivel bancario y apoyos económicos a las naciones en desarrollo.

Para cumplir con sus labores el Grupo del Banco Mundial cuenta con un equipo de apoyo que se expondrá a continuación (Banco Mundial, 2005):

El Banco Internacional de Reconstrucción y Fomento (BIRF, 185 países miembros ${ }^{17}$ ), cuyo objetivo es reducir la pobreza en los países en vía de desarrollo y de mediano ingreso con capacidad crediticia.

La Asociación Internacional del Fomento (AIF), proporciona dinero para la construcción de servicios básicos como educación, vivienda, agua potable, entre

\footnotetext{
${ }^{16}$ Relación entre monedas de varios países, o entre su valor monetario y su peso en metal; igualdad de las cosas entre sí.

${ }^{17}$ Está integrado por los mismos países que conforman el FMI.
} 
otros, generando reformas e inversiones para fomentar el aumento del empleo y la productividad.

La Corporación Financiera Internacional (CFI), se encarga de promover el desarrollo económico de los países a través del sector privado, es decir, los socios comerciales invierten capital por medio de empresas privadas en los países en desarrollo.

El Organismo Multilateral de Garantía de Inversiones (OMGI), su función es promover la inversión extranjera en países subdesarrollados, proporcionando garantías a los inversionistas contra pérdidas ocasionadas por riesgos no comerciales.

Y el Centro Internacional de Arreglo de Diferencias Relativas a Inversiones (CIADI), tiene como meta cuidar la inversión extranjera en los países, sirviendo de conciliadores y órgano arbitrario de diferencias entre los implicados.

\section{¿Por qué han tenido inconvenientes el Fondo Monetario Internacional y el Banco Mundial?}

Todos los propósitos, metas y visiones a cumplir, tanto por el Banco Mundial como del Fondo Monetario Internacional son interesantes al momento de ser plasmados en un papel o publicados en una página web, pero a la hora de la verdad esos propósitos establecidos en un principio se empezaron a ejecutar por poco tiempo. Esto debido a que los países desarrollados pusieron los ojos en los países en vía de desarrollo y cambiaron totalmente su objetivo. Es decir, lograron engañar a los africanos y especialmente a los americanos, porque dejaron que el Fondo Monetario Internacional interviniera en la economía de sus países y los dejaran en una peor situación.

De la misma manera, el Banco Mundial supuestamente contribuye a reducir la pobreza en estos países, pero no es así, porque pese a que mencionan que les ofrecen préstamos con bajas tasas de interés, con qué dinero van a pagar la deuda si no existen recursos para responder. Es por eso, que el Grupo del Banco Mundial, primero debe estudiar el caso de cada país para ver si les dan ese crédito 
ACTIVOS | REVISTA DE LA FACULtad de CONTADURía PúBlica

$\mathrm{y}$, segundo, observar con qué le pueden respaldar esa deuda en caso de no tener liquidez en el momento de cancelar la obligación.

Pero quizás, el problema más grave que tienen estos organismos es que Estados Unidos interviene en todo, y no es que sean inventos de la gente sino que por el contrario, los hechos hablan por sí solos. Se hace por ejemplo, referencia a la situación que está viviendo Irán en donde éste se ha quejado con el FMI sobre tentativas de Estados Unidos de aislar financieramente la República Islámica.

De igual forma, este imperio -Estados Unidos- se ha atrevido a sancionar a bancos como el Sepah y el Saderat y está presionando a otros cuantos por hacer negocios con Irán. Todo esto lo hacen, según ellos, porque los bancos sirven a compañías implicadas en el programa nuclear de Irán (MEED, 2007, p. 1).

Otro ejemplo claro que es importante citar es el que vivió el BM por el escándalo de su anterior presidente Paul Wolfowitz al darle una generosa compensación a una conocida suya. Este hecho causó gran controversia durante un mes, ya que amenazó con el desprestigio de esta institución que lucha por erradicar la pobreza, y de su habilidad de convencer a los países alrededor del mundo para contribuir con los mil millones de dólares que proporcionan ayuda financiera a naciones pobres.

La anterior situación, se debió a que al Señor Wolfowitz le dieron mucha libertad porque quien lo eligió fue el actual presidente de los Estados Unidos, George W. Bush en el 2005, de hecho, por tradición el jefe del BM es escogido por el presidente de los estadounidenses. Afortunadamente, el escándalo no pasó a mayores, en cuanto al Banco Mundial se refiere, pero por desgracia su presidente no aguantó más y trabajó hasta el pasado 30 de junio del año 2007, cuando presentó su renuncia.

Este tipo de situaciones se debe a la hegemonía que tiene Estados Unidos en estas instituciones. En la actualidad 185 países integran el Fondo Monetario Internacional. De éstos, 178 países no tienen ni voz ni voto (Fondo Monetario Internacional, 2001).

Tabla 1. Países miembros del FMI 


\begin{tabular}{|c|c|c|c|c|}
\hline $\begin{array}{l}\text { Afganistán, } \\
\text { Albania, } \\
\text { Algeria, Angola, } \\
\text { Antigua y } \\
\text { Barbuda, Arabia } \\
\text { Saudita, } \\
\text { Argentina, } \\
\text { Armenia, } \\
\text { Australia, } \\
\text { Austria, } \\
\text { Azerbaiyán }\end{array}$ & $\begin{array}{l}\text { Bahamas, } \\
\text { Bahrein, } \\
\text { Bangladesh, } \\
\text { Barbados, } \\
\text { Belarús, } \\
\text { Bélgica, Belice, } \\
\text { Benin, Bhután, } \\
\text { Bolivia, Bosnia } \\
\text { y Herzegovina, } \\
\text { Botswana, } \\
\text { Brasil, } \\
\text { Brunei Darus- } \\
\text { salam, Bulgaria, } \\
\text { Burkina Faso, } \\
\text { Burundi }\end{array}$ & $\begin{array}{l}\text { Camboya, Cabo } \\
\text { Verde, Camerún } \\
\text { Canadá, Chad, } \\
\text { Chile, China, } \\
\text { Colombia, } \\
\text { Comoros, Costa } \\
\text { Rica, Cote de } \\
\text { Marfil, Congo, } \\
\text { República } \\
\text { Demo- } \\
\text { crática } \\
\text { Congo, del } \\
\text { República de } \\
\text { Corea, } \\
\text { República de } \\
\text { Croacia, } \\
\text { Cyprus, } \\
\text { República Checa }\end{array}$ & $\begin{array}{l}\text { Dinamarca, } \\
\text { Djibouti, } \\
\text { Dominica, } \\
\text { Ecuador, } \\
\text { Egipto, El } \\
\text { Salvador, } \\
\text { Emiratos Árabes } \\
\text { Unidos, Eritrea, } \\
\text { Eslovenia, } \\
\text { España, Estados } \\
\text { Unidos, Estonia, } \\
\text { Etiopía, Federa- } \\
\text { ción de Rusia, } \\
\text { Fiji, Finlandia, } \\
\text { Francia }\end{array}$ & $\begin{array}{l}\text { Gabon, Gambia, } \\
\text { Georgia, Alema- } \\
\text { nia, Ghana, } \\
\text { Grecia, } \\
\text { Grenada, } \\
\text { Guatemala, } \\
\text { Guinea, Guinea- } \\
\text { Bissau, Guinea } \\
\text { Ecua- } \\
\text { torial, Guyana, } \\
\text { Haití, Honduras, } \\
\text { Hungría, } \\
\text { Islandia, } \\
\text { India, Indonesia, } \\
\text { República } \\
\text { Islámica de Irán, } \\
\text { Iraq, Irlanda, } \\
\text { Israel, } \\
\text { Italia }\end{array}$ \\
\hline $\begin{array}{l}\text { Jamaica, Japón, } \\
\text { Jordania, } \\
\text { Kazajstán, } \\
\text { Kenya, Kiribati, } \\
\text { Kuwait, Laos, } \\
\text { Letonia, Líbano, } \\
\text { Lesotho, } \\
\text { Liberia, Libia, } \\
\text { Lituania, } \\
\text { Luxembourgo, } \\
\text { Macedonia, Ma- } \\
\text { dagascar, } \\
\text { Malawi, } \\
\text { Malasia, } \\
\text { Maldivas, Mali, } \\
\text { Malta, } \\
\text { Marshall, Islas } \\
\text { Mauritania, } \\
\text { Mauricio, } \\
\text { México, Estados } \\
\text { Unidos } \\
\text { Micronesia, de } \\
\text { Moldova, } \\
\text { Mongolia, } \\
\text { Montenegro, } \\
\text { Marruecos, } \\
\text { Mozambique, } \\
\text { Myanmar }\end{array}$ & $\begin{array}{l}\text { Namibia, Ne- } \\
\text { pal, Países } \\
\text { bajos, } \\
\text { Nueva Zelanda, } \\
\text { Nicaragua, } \\
\text { Níger, Nigeria, } \\
\text { Noruega, Omán, } \\
\text { Pakistán, Palau, } \\
\text { Panamá, Papua, } \\
\text { Nueva } \\
\text { Guinea, } \\
\text { Paraguay, } \\
\text { Perú, Filippinas, } \\
\text { Polonia, } \\
\text { Portugal } \\
\text { Qata }\end{array}$ & $\begin{array}{l}\text { Reino Unido, } \\
\text { República } \\
\text { Árabe, Siria, } \\
\text { República } \\
\text { Centroafricana, } \\
\text { República } \\
\text { Dominicana, } \\
\text { República de } \\
\text { Kirguistán, } \\
\text { Rumania, } \\
\text { Rwanda }\end{array}$ & $\begin{array}{l}\text { Samoa, San } \\
\text { Marino, Santo } \\
\text { Tomé } \\
\text { Príncipe, } \\
\text { Senegal, Serbia, } \\
\text { Seychelles, } \\
\text { Sierra Leona, } \\
\text { Singapur, } \\
\text { República } \\
\text { Eslovaca, Islas } \\
\text { Salomón, Soma- } \\
\text { lia, Sudáfrica, } \\
\text { Sri Lanka, St. } \\
\text { Kitts y Nevis, } \\
\text { St. Lucía, St. } \\
\text { Vicente y las } \\
\text { Granadinas, } \\
\text { Sudán, } \\
\text { Suriname, } \\
\text { Swaziland, } \\
\text { Sweden, } \\
\text { Switzerlandia, }\end{array}$ & $\begin{array}{l}\text { Tayikistán, } \\
\text { Tanzania, } \\
\text { Tailandia, } \\
\text { Timor-Leste, } \\
\text { Togo, Tonga, } \\
\text { Trinidad y } \\
\text { Tobago, Túnez, } \\
\text { Turquía, } \\
\text { Turkmenistán, } \\
\text { Uganda, } \\
\text { Ucraina, Uru- } \\
\text { guay, } \\
\text { Uzbekistán, } \\
\text { Vanuatu, Repú- } \\
\text { blica } \\
\text { Bolivariana de } \\
\text { Venezuela, Viet } \\
\text { Nam, República } \\
\text { del Yemen, } \\
\text { Zambia, } \\
\text { Zimbabwe. }\end{array}$ \\
\hline
\end{tabular}


Fuente: Fondo Monetario Internacional.

Lo anterior, se debe a que las naciones que aportan las mayores contribuciones monetarias al Fondo Común son las de más poder en la toma de decisiones. En el Consejo de Gobernadores, el máximo gremio de dicha institución, Estados Unidos tiene el 17,1\% de los votos, con lo cual adquiere el "control accionario" $\mathrm{y}$, con ello, el derecho a veto, en vista de que las decisiones pueden ser tomadas sólo con una mayoría calificada del $85 \%$.

Las naciones acreedoras del G-7 controlan las decisiones del directorio ejecutivo. Cinco de ellas (EE.UU, Japón, Alemania, Francia y Gran Bretaña), con más del $35 \%$ de los votos, son nombradas en el directorio, por lo que el FMI ha sido definido, un tanto irónicamente, como "la instancia neutral de los acreedores" (ver Figura 1). Si se observan estos porcentajes para nadie es un secreto la hegemonía que tiene el imperio estadounidense no sólo sobre los africanos sino también hacia sus vecinos, América Latina.

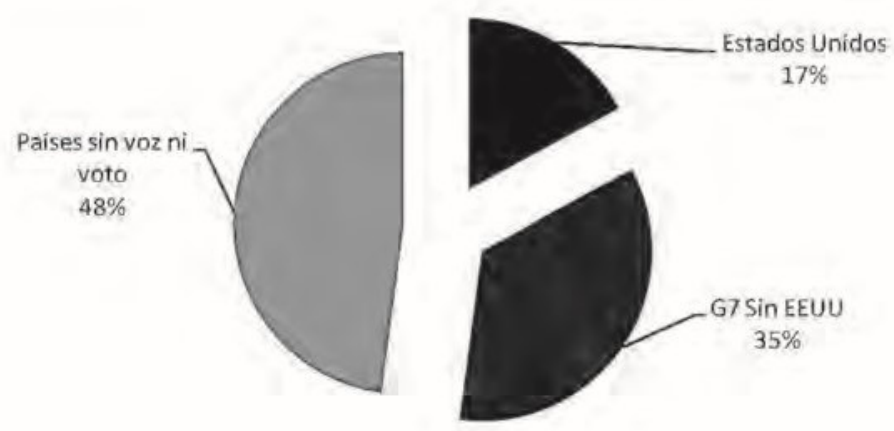

Figura 1. Control de votos en la Junta Ejecutiva del FMI.

Fuente: Elaboración propia.

De igual manera, todos los componentes del Grupo del Banco Mundial son propiedad de los países miembros. Cuando un país se incorpora al Banco garantiza una suscripción de capital, pagando sólo un pequeño porcentaje de dicha garantía. El resto del dinero es pagadero a la vista y sirve de garantía blindada, es decir, asegura el pago de la deuda.

La suscripción de capital asignada es proporcional a la riqueza del país determinando el número de votos de cada uno de éstos: por ejemplo, EE.UU. 
controla el 16,39\% de los votos; Japón el 7,86\%, Alemania 4,49\%, Francia 4,30\% y Gran Bretaña el 4,30\%. En contraste 24 países africanos controlan juntos sólo el 2,85\% del total (ver Tabla 2).

Tabla 2. Poder de voto en el BM

\begin{tabular}{|l|c|}
\hline \multicolumn{1}{|c|}{ Poder } & Porcentaje \\
\hline Estados Unidos & $16,39 \%$ \\
\hline Japón & $7,86 \%$ \\
\hline Alemania & $4,49 \%$ \\
\hline Francia & $4,30 \%$ \\
\hline Reino Unido & $4,30 \%$ \\
\hline Italia & $4,30 \%$ \\
\hline Otros & $58,36 \%$ \\
\hline
\end{tabular}

Para muchos, estos organismos son de vital importancia para el desarrollo económico, político y social. Esto es innegable, pero lo que está escrito y plasmado en tratados no quiere decir que se va a cumplir.

\section{¿Qué se puede hacer?}

Para darle solución a todos esos aspectos negativos, fallas y deficiencias que se están presentando en el Fondo Monetario Internacional y en el Banco Mundial es necesaria una reforma urgente en la Arquitectura Financiera Internacional.

Es por esto que se habla de la Nueva Arquitectura Financiera Internacional (NAFI) tal como lo plantea Kelyneth Peña (2007) se define como:

... el conjunto orgánico, que exige la aplicación de un enfoque integral, que debe comprender en ese mismo conjunto ciertos elementos interrelacionados relativos al manejo de la liquidez internacional, las regulaciones financieras, las políticas macroeconómicas que deberá extenderse al financiamiento para el desarrollo y solucionar los problemas 
ACTIVOS | REVISTA de la FaCultad de contaduría pública

de la deuda externa y finalmente dar bases consistentes para evitar, controlar las crisis.

Los países desarrollados tienen diferentes propuestas para corregir la arquitectura financiera como es el caso del gobierno francés, quien le da un poder adicional para la toma de decisiones al Comité Interino de Ministros de Finanzas. El gobierno alemán ha puesto a discusión la idea de zonas concretas para tipos de cambios, que permitirían evitar el mal comportamiento de las monedas. Y en el caso del gobierno canadiense, se propone una sanción al Fondo Monetario Internacional o una detención de pagos si se presentaran dificultades financieras (Eichengreen, 2000, p.1-41).

Para Barry Eichengreen (2000) deben existir tres dispositivos para mejorar el sistema financiero: prevención de las crisis, predicción de las crisis y administración de las crisis.

En el primero se debe mejorar la información sobre los asuntos económicos y financieros de los gobiernos, bancos y empresas para fortalecer la disciplina del mercado y ayudar a quienes elaboran las políticas económicas a identificar la necesidad de acciones correctivas; además, la estabilización del sistema financiero necesita reformas institucionales que se extiendan mucho más allá de la política, hacia el comercio exterior y los pagos.

En el segundo dispositivo, por más que se prevengan las crisis, éstas seguirán ocurriendo debido a la incursión de nueva información, nuevas interpretaciones, la salida de inversionistas potenciales de la economía de un país, no seguir cotizando bolsa, entre otros factores.

Y en el tercer dispositivo, es tal vez en el cual se presentan las mayores falencias en la arquitectura financiera internacional, esto debido a que la comunidad mundial tiene dos maneras de actuar cuando se exteriorizan las crisis; una es, correr al rescate del país en crisis con una bolsada llena de dinero y la otra es, hacer de cuenta que nada ha pasado y dejar que continúe el problema.

De la misma manera, el Grupo de Trabajo del Comité Ejecutivo de Asuntos Económicos y Sociales de las Naciones Unidas (1999) menciona que las instituciones actuales no son las adecuadas para enfrentar la globalización, esto 
debido a la enorme discrepancia que existe entre un mundo financiero internacional cada vez más sofisticado y dinámico, con rápida globalización de las carteras financieras, y la ausencia de un marco institucional capaz de regularlo.

También argumentan que el Fondo Monetario Internacional debe crear fondos para contingencias y que asistan a los países actualmente afectados o contagiados por las crisis, y también a los que puedan convertirse en el futuro en víctimas de las crisis financieras mundiales. Para ello, el Grupo de los Siete ha tomado algunas medidas para garantizar el adecuado financiamiento de contingencia, con el aumento de las cuotas del Fondo, los nuevos acuerdos para la obtención de préstamos y el compromiso de complementar los recursos del Fondo Monetario Internacional según sea el caso.

Pero en realidad lo que se debe hacer con extrema urgencia es lograr que estos organismos replanteen sus objetivos y metas, y en especial, amplíen sus horizontes, es decir, que el Grupo de los Siete no sea limitado sino que al contrario sea un grupo en el cual participen tanto países desarrollados como subdesarrollados.

De igual manera, si se reforma la arquitectura financiera internacional, se debe empezar por no poner tantas condiciones ni exigir tantos requisitos para poder acceder a préstamos u otros beneficios que éstos otorgan, al igual que su futuro cobro; por ejemplo, si el país al que se le otorgó el crédito no tiene manera de pagar éste, en el momento en que se haga efectivo su cobro estos organismos pueden proceder a la enajenación de bienes inmuebles y muebles, y ha entablar procedimientos legales, en contra de ese país.

Igualmente, el FMI y el BM deben hacer que los créditos que se dan a los países en vía de desarrollo no sean tan bajos porque sus necesidades sociales son gigantescas y además, el mundo se encuentra en la era de la globalización en el cual se debe modernizar y unificar primero el comercio intraregional y luego el comercio exterior; un ejemplo claro de esto es el Líbano que tiene un expediente impecable por pagar sus obligaciones a tiempo, mientras que el FMI le presta a éste una proporción muy pequeña con referencia a la capacidad que tiene éste de compensar, según lo medido por sus reservas internacionales [más de \$11000 
ACTIVOS | REVISTA de la FaCultad de contaduría pública

millones] o sus exportaciones [más de $\$ 13000$ millones] (Middle East Economic Digest, 2007).

\section{Conclusiones}

Es de gran relevancia que la Nueva Arquitectura Financiera Internacional se lleve a cabo, pero en favor del desarrollo económico, político y social en el mundo; es decir, para que exista este progreso tanto el Banco Mundial como el Fondo Monetario Internacional deben en primera instancia, crear un plan estratégico, independiente el uno del otro, para evitar causar o terminar de hundir a los países que están ayudando en momentos de crisis ya sean económicos o sociales; y en segunda instancia, se debe crear un organismo que tenga autoridad financiera mundial quien dicte normas internacionales necesarias para supervisar y regularizar la situación financiera global.

Con todo esto queda claro que si el FMI y el BM siguen de la mano del Imperio, sus objetivos como organismos de apoyo se van a desviar y van a resultar haciendo lo que le convenga a los Estados Unidos y el esfuerzo que se hizo de reformar la arquitectura financiera internacional no habrá servido de nada.

\section{Referencias}

Banco Mundial (2005). es.wikipedia.org/Banco_Mundial. Extraído el 27 de mayo de 2007.

Business week online (2007). Wolfowitz out in June at World Bank, 1.

Cavanaugh J. et al. (1994). Alternativas al orden económico global. Barcelona: ICAIRA Editorial. S.A.

Central Europe \& Baltics (2007). IMF expresses doubts about anti - inflation plan. London.

Cuevas, V. et al. (2006). El FMI y la nueva arquitectura financiera mundial.Bogotá: Red Agrociencia. 
Eichengreen, B. (2000). Hacia una nueva arquitectura financiera internacional. México: Oxford.

Fondo Monetario Internacional (2001). www.choike.org, consultado el 27 de mayo de 2007.

Emst, J. (2007). The World Bank's real problema. New York: New York Times. (p. 1)

Informe del Grupo de Trabajo del Comité Ejecutivo de Asuntos Económicos y Sociales de las Naciones Unidas (1999). Hacia una nueva arquitectura financiera internacional. www.geocities.com/perfilgerencial/arquitecturafinanciera.html. Extraído el 27 de mayo de 2007.

Kovac, M. (2007). IMF dobouts GCC currency union. ICIS chemical business, $2,(1)$.

MEED. (2007). Tehran petitions IMF on US. Extraído de http://www.accessmylibrary.com/

Middle east economic digest (2007). IMF loan carries fiscal reform conditions. Extraído de http://www.accessmylibrary.com/

The International Monetary Fund Publications (2006). The state of state reform in latin american. Extraído el 27 de mayo de 2007. www.worldbank.com.

Extraído en julio de 2007. 